\title{
MICROPROCESSES AT SECOND-ORDER PHASE TRANSITIONS IN CRYSTALS WITH STRONG INTERBAND ELECTRON-PHONON INTERACTION
}

\author{
E.N. MYASNIKOV, Z.P. MASTROPAS
}

PACS 64.60.Bd

(C) 2011

\author{
Southern Federal University \\ (Rostov-on-Don, Russia; e-mail: mastrozin@mail.ru)
}

\begin{abstract}
A system of strongly interacting electrons and phonons in a crystal has been considered. If the temperature changes, the system undergoes changes equivalent to those occurring at the phase transition of the second kind, when the equilibrium positions of atoms become shifted. It has been demonstrated that the expansion of the thermodynamic potential in a series in the order parameter, which is a standard routine in the Landau phenomenological theory, can lead to equilibrium states that do not correspond to any real state of the crystal. It has also been shown that, in the course of phase transitions that occur with varying temperature, the deformation energy is released in the form of Barkhausen-like pulses.
\end{abstract}

\section{Introduction}

It was proved by V.L. Bonch-Bruevich long ago that a crystal is thermodynamically equivalent to the system of its elementary excitations (quasiparticles) [1]. Therefore, provided that the properties of the quasiparticle system in the crystal are determined experimentally at a certain temperature, we can take advantage of the powerful methods of the quantum field theory to find the properties of the system at any other temperature and, therefore, to come to know how the properties of the crystal change with the temperature. The band-to-band electron-phonon interaction brings about a variation of the Bloch amplitudes for valence electrons through the hybridization of their wave functions with the wave functions of conduction electrons. As a result, the distribution of valence electrons in the crystal unit cell changes in such a way that, taking the energy of electron-phonon coupling into account, the total energy of the system decreases, and the crystal structure (more specifically, the average equilibrium positions of the atoms) changes. Such a scenario was used for the first time to consider the ferroelectric phase transition in crystals of the $\mathrm{BaTiO}_{3}$ type [2]. The results obtained (the temperature of the transition, the values calculated for the parameters in the Curie-Weiss law and the "law of two") turned out in good agreement with experimen- tal data. Within the method of temperature (Matsubara) Green's functions, we calculated [3] a correction to the equilibrium thermodynamic potential for a crystal of the $\mathrm{BaTiO}_{3}$ type, which emerges owing to the bandto-band interaction of electrons with transverse optical phonons. The correction turned out to diverge logarithmically as $\ln \frac{T-T_{c}}{T_{c}}$, when approaching the point of the ferroelectric phase transition of the second kind (FT2), $T_{c}$. The same divergence was revealed while studying the equilibrium thermodynamic potential of the crystal that undergoes a ferroelastic phase transition owing to the interband interaction of electrons with transverse acoustic phonons.

The FT2 takes also place in a two-dimensional Ising lattice. Onsager [4] managed to obtain an exact form for the equilibrium thermodynamic potential of the Ising lattice, which looks like a sum that includes a diverging term; the latter also has a logarithmic singularity of the type $\ln \frac{T-T_{c}}{T_{c}}$ at $T \rightarrow T_{c}$ in the interval $T \geq T_{c}$. The symmetry of the systems considered in the three indicated examples changes at the FT2 point, so that, as was stated in work [4], the diverging term has to exist at $T>T_{c}$, but it has no meaning on the other side of the phase transition point (at $T<T_{c}$ ). Really, the expression $\ln \frac{T-T_{c}}{T_{c}}$ cannot exist at $T<T_{c}$. Hence, the indicated logarithmic singularity satisfies all the attributes typical of a singularity in the equilibrium thermodynamic potential at the FT2 point. On this basis, we adopt that the equilibrium thermodynamic potential has a corresponding logarithmic singularity at an arbitrary FT2.

The phenomenological theory of FT2 created by Landau [5] is constructed on the basis of the assumption that the temperature- and pressure-dependent coefficient $A$ before the squared order parameter, $\eta^{2}$, in the expansion of the thermodynamic potential $\Phi(P, T)$ in a power series of $\eta$ is proportional to the difference $T-T_{c}$. The thermodynamic potential with such a coefficient is not equilibrium, and the equilibrium condition $\frac{\Phi}{\eta}=0$ can be not associated with any real macroscopic state of the 
system. A doubt remains only with respect to the equilibrium condition at the phase transition point, $T=T_{c}$.

"We must emphasize, however, that such states do in fact exist near a phase transition point of the second kind: as the transition point is approached, the minimum of $\Phi$ as a function of $\eta$ becomes steadily flatter. This means that the "restoring force" that tends to bring the body to the state having the equilibrium value of $\eta$ becomes steadily weaker, so that the relaxation time for the establishment of the equilibrium with respect to the order parameter increases without limit (and certainly becomes much longer than the time for equalization of the pressure throughout the body)" [5]. This reasoning seems to consider the $\eta$-value obtained from the condition $\frac{\Phi}{\eta}=0$ to be practically equilibrium only at $T=T_{c}$. However, since the potential $\Phi$ can probably be regarded as equilibrium at $A \sim \ln \frac{T-T_{c}}{T_{c}}$, the solution of the equation $\frac{\Phi}{\eta}=0$ for the equilibrium potential gives us a correct equilibrium value for $\eta$, as well as a probable real macroscopic state of the body, at any temperature $T>T_{c}$. Moreover, the "restoring force" does not tend to zero in the case of a potential with logarithmic divergence, and the nonequilibrium potential cannot be used to determine the order parameter as the ratio $\frac{\Phi}{\eta}$ even at $T \rightarrow T_{c}$.

Thus, while approaching the FT2 point as the temperature decreases in the interval $T>T_{c}$, the equilibrium thermodynamic potential tends to infinity. At the same time, it cannot be calculated in the interval $T<T_{c}$, i.e. in the low-temperature phase, because the FT2 gives rise to a reconstruction of the vibration mode branches and a change of the crystal symmetry. The change over to a new system of normal coordinates for crystal lattice vibrations considerably complicates the procedure of finding $\Phi(P, T)$.

\section{Crystal Energy at $T \rightarrow 0$}

An ordered phase can be analyzed at $T=0$ as a system of quasiparticles by calculating its average energy, because its entropy vanishes in this case.

The Hamiltonian of the system is written down in its ordinary form for electron-phonon systems (hereafter, $\hbar=1)$,

$$
\begin{aligned}
& \hat{H}=\sum_{\mathbf{k}, \sigma}^{2} E_{\sigma}(\mathbf{k}) a_{\sigma \mathbf{k}}^{+} a_{\sigma \mathbf{k}}+\sum_{\mathbf{q}} \omega(\mathbf{q}) b_{\mathbf{q}}^{+} b_{\mathbf{q}}+ \\
& +\sum_{\mathbf{k}, \mathbf{q} ; \sigma^{\prime} \neq \sigma=1}^{2} N^{-1 / 2} \sqrt{\frac{\omega(\mathbf{q})}{2}} \times
\end{aligned}
$$

$\times \Gamma_{\sigma \sigma^{\prime}}(\mathbf{k}, \mathbf{k}-\mathbf{q}) a_{\mathbf{k} \sigma}^{+} a_{\mathbf{k}-\mathbf{q}, \sigma^{\prime}}\left(b_{\mathbf{q}}+b_{-\mathbf{q}}^{+}\right)$,

where $a_{\sigma \mathbf{k}}^{+}$and $a_{\sigma \mathbf{k}}$ are the creation and annihilation, respectively, operators of an electron with the wave vector $\mathbf{k}$ in the $\sigma$-th band ( $\sigma=1$ for the valence band and 2 for the conduction one), and $b_{\mathbf{q}}^{+}$and $b_{\mathbf{q}}$ are the creation and annihilation, respectively, operators for phonons. Let us apply a unitary transformation to the phonon creation and annihilation operators in Eq. (1). This operation enables the sum (with corresponding coefficients) of average values of the coordinate, $q$, and the momentum, $p$, for the $\mathbf{k}$-th lattice vibration harmonic to be singled out into a separate classical variable $d_{\mathbf{k}}$,

$U(d)=\prod_{\mathbf{k}} \exp \left(d_{\mathbf{k}} b_{\mathbf{k}^{\prime}}^{+}-d_{\mathbf{k}}^{*} b_{\mathbf{k}^{\prime}}\right)=$

$=\prod_{\mathbf{k}} \exp \left(\frac{i}{\hbar}\left(p_{\mathbf{k}} \hat{Q}_{\mathbf{k}^{\prime}}-q_{\mathbf{k}} \hat{P}_{\mathbf{k}^{\prime}}\right)\right)$.

With this transformation, new phonon operators are $b_{\mathbf{k}}^{\prime}=U b_{\mathbf{k}} U^{-1}=b_{\mathbf{k}}-d_{\mathbf{k}}$ and $b_{\mathbf{k}}^{+}=U b_{\mathbf{k}}^{+} U^{-1}=b_{\mathbf{k}}^{+}-d_{\mathbf{k}}^{*}$, so that the average value for the operator $b_{\mathbf{k}}^{\prime}$ equals zero. After carrying out this unitary transformation, Hamiltonian (1) looks like

$$
\begin{aligned}
& \hat{H}^{\prime}=U \hat{H} U^{-1}=\sum_{\mathbf{k}, \sigma=1}^{2} E_{\sigma}(\mathbf{k}) a_{\sigma \mathbf{k}}^{+} a_{\sigma \mathbf{k}}+\sum_{\mathbf{q}} \omega(\mathbf{q}) b_{\mathbf{q}}^{\prime+} b_{\mathbf{q}}^{\prime}+ \\
& +\sum_{\mathbf{k}, \mathbf{q} ; \sigma \neq \sigma^{\prime}=1}^{2} \sqrt{\frac{\omega(\mathbf{q})}{2 N}} \mathbf{g}_{0}(\mathbf{q}) a_{\mathbf{k} \sigma}^{+} a_{\mathbf{k}-\mathbf{q}, \sigma^{\prime}}\left(b_{\mathbf{q}}^{\prime}+b_{\mathbf{q}}^{\prime+}\right)
\end{aligned}
$$

Using the transformation rules indicated above for the operators $b_{\mathbf{k}}^{\prime}$ and ${b_{\mathbf{k}}^{\prime}}_{\mathbf{k}}^{+}$, Hamiltonian (3) can be expressed in the form

$$
\begin{aligned}
& \hat{H}^{\prime}=\sum_{\mathbf{k}, \sigma}^{2} E_{\sigma}(\mathbf{k}) a_{\sigma \mathbf{k}}^{+} a_{\sigma \mathbf{k}}+\sum_{\mathbf{q}} \omega(\mathbf{q}) b_{\mathbf{q}}^{+} b_{\mathbf{q}}+ \\
& +\sum_{\mathbf{k}, \mathbf{q} ; \sigma^{\prime} \neq \sigma=1}^{2} \sqrt{\frac{\omega(\mathbf{q})}{2 N}} \mathbf{g}_{0}(\mathbf{q}) a_{\mathbf{k} \sigma}^{+} a_{\mathbf{k} \sigma}\left(b_{\mathbf{q}}+b_{-\mathbf{q}}^{+}\right)+\hat{H}^{\prime \prime}
\end{aligned}
$$$$
\hat{H}^{\prime \prime}=\sum_{\mathbf{q}} \omega(\mathbf{q})\left(d_{\mathbf{q}}^{*} d_{\mathbf{q}}-b_{\mathbf{q}}^{*} d_{\mathbf{q}}-d_{\mathbf{q}}^{*} b_{\mathbf{q}}\right)-
$$$$
-\sum_{\mathbf{k}, \mathbf{q} ; \sigma^{\prime} \neq \sigma} \sqrt{\frac{\omega(\mathbf{q})}{2 N}} \mathbf{g}_{0}(\mathbf{q}) a_{\mathbf{k} \sigma}^{+} a_{\mathbf{k}-\mathbf{q}, \sigma^{\prime}}\left(d_{\mathbf{q}}+d_{-\mathbf{q}}^{*}\right) .
$$ 
For the sake of simplicity, let us firstly determine, using the variational method, the ground state of the system, in which the average value of the Hamiltonian $\hat{H}=\hat{H}^{\prime}-\hat{H}^{\prime \prime}$ is minimal. As a varied vector of the ground state, we use the vector

$$
|d, \psi\rangle \equiv \psi \exp \left\{\sum_{\mathbf{q}}\left(d_{\mathbf{q}} b_{\mathbf{q}}^{+}-d_{\mathbf{q}}^{*} b_{\mathbf{q}}\right)\right\}|0\rangle=\psi U(d)|0\rangle,
$$

in which $|0\rangle$ is the vector of state for the phonon field free of phonons and without vacuum deformation, and the varied parameters $d_{\mathbf{q}}$ coincide with those contained in expression (2). The wave function $\psi$ of the electron subsystem in the secondary quantization representation will be determined later together with its varied parameters. Now, let us determine the average value of the operator $\hat{H}=\hat{H}^{\prime}-\hat{H}^{\prime \prime}$ in the state $|d, \psi\rangle$. We should take into account that $\left\langle d, \psi\left|\hat{H}^{\prime}\right| d, \psi\right\rangle=\sum_{\mathbf{k}, \sigma=1}^{2} E_{\sigma}(\mathbf{k})\left\langle\psi\left|a_{\sigma \mathbf{k}}^{+} a_{\sigma \mathbf{k}}\right| \psi\right\rangle$, because $\left\langle 0\left|b_{\mathbf{q}}^{\prime+} b_{\mathbf{q}}^{\prime}\right| 0\right\rangle=0$ and $\left\langle 0\left|b_{\mathbf{q}}^{\prime}\right| 0\right\rangle=\left\langle 0\left|{b_{\mathbf{q}}^{\prime}}_{\mathbf{q}}^{+}\right| 0\right\rangle=0$. Similarly, using the relations $\left\langle d, \psi\left|b_{\mathbf{q}}\right| d, \psi\right\rangle=d_{\mathbf{q}}$ and $\left\langle d, \psi\left|b_{\mathbf{q}}^{+}\right| d, \psi\right\rangle=d_{\mathbf{q}}^{*}$, we obtain

$$
\begin{aligned}
& \left\langle d, \psi\left|\hat{H}^{\prime \prime}\right| d, \psi\right\rangle=-\sum_{\mathbf{q}}\left\{\omega(\mathbf{q}) d_{\mathbf{q}}^{*} d_{\mathbf{q}}+\right. \\
& \left.+\sum_{\mathbf{k}, \sigma \neq \sigma^{\prime}} \sqrt{\frac{\omega(\mathbf{q})}{2 N}} \mathbf{g}_{0}(\mathbf{q})\left\langle\psi\left|a_{\mathbf{k} \sigma}^{+} a_{\mathbf{k}-\mathbf{q}, \sigma^{\prime}}\right| \psi\right\rangle\left(d_{\mathbf{q}}+d_{-\mathbf{q}}^{+}\right)\right\} .
\end{aligned}
$$

Hence, it turns out that

$$
\begin{aligned}
& \langle d, \psi|\hat{H}| d, \psi\rangle=\sum_{\mathbf{k}, \sigma=1}^{2} E_{\sigma}(\mathbf{k})\left\langle\psi\left|a_{\sigma \mathbf{k}}^{+} a_{\sigma \mathbf{k}}\right| \psi\right\rangle+ \\
& +\sum_{\mathbf{q}}\left\{\omega(\mathbf{q}) d_{\mathbf{q}}^{*} d_{\mathbf{q}}+\sum_{\mathbf{k}, \sigma \neq \sigma^{\prime}} \sqrt{\frac{\omega(\mathbf{q})}{2 N}} \times\right. \\
& \left.\times \mathbf{g}_{0}(\mathbf{q})\left\langle\psi\left|a_{\mathbf{k} \sigma}^{+} a_{\mathbf{k}-\mathbf{q}, \sigma^{\prime}}\right| \psi\right\rangle\left(d_{\mathbf{q}}+d_{-\mathbf{q}}^{*}\right)\right\} .
\end{aligned}
$$

Since the quantity

$$
\bar{u}_{\mathbf{q}}=\sqrt{\frac{1}{2} M \omega(\mathbf{q})}\left\langle\psi\left|b_{\mathbf{q}}+b_{\mathbf{q}}^{+}\right| \psi\right\rangle=\sqrt{\frac{1}{2} M \omega(\mathbf{q})}\left(d_{\mathbf{q}}+d_{\mathbf{q}}^{*}\right)
$$

must be real-valued, it has to be that $d_{\mathbf{q}}=d_{-\mathbf{q}}$. Making the substitution $d_{\mathbf{q}}=\left|d_{\mathbf{q}}\right| \exp \left(i \varphi_{\mathbf{q}}\right)$ and zeroing the derivative of function (6) with respect to $\left|d_{\mathbf{q}}\right|$, we determine the corresponding extreme $\left|d_{\mathbf{q}}\right|$-value,

$$
\begin{aligned}
& \langle d, \psi|\hat{H}| d, \psi\rangle=\sum_{\mathbf{k}, \sigma=1}^{2} E_{\sigma}(\mathbf{k})\left\langle\psi\left|a_{\sigma \mathbf{k}}^{+} a_{\sigma \mathbf{k}}\right| \psi\right\rangle- \\
& -\sum_{\mathbf{q}} \omega(\mathbf{q})\left|d_{\mathbf{q}}\right|^{2} \\
& \left|d_{\mathbf{q}}\right|=-\frac{\cos \varphi(\mathbf{q})}{\sqrt{2 N \omega(\mathbf{q})}} \sum_{\mathbf{k}, \sigma \neq \sigma^{\prime}} \mathbf{g}_{0}(\mathbf{q})\left\langle\psi\left|a_{\sigma \mathbf{k}}^{+} a_{\sigma^{\prime}, \mathbf{k}-\mathbf{q}}\right| \psi\right\rangle .
\end{aligned}
$$

Therefore, at the strong enough electron-phonon coupling, the lattice deformation energy turns out, by magnitude, half as much as the negative energy of interaction between electrons and the lattice deformation.

Since the $d_{\mathbf{q}}$-value must be positive, the minimum value of the Hamiltonian $\hat{H}$ averaged over the variable $\varphi_{\mathbf{q}}$ is attained at $\cos \varphi_{\mathbf{q}}=-1$, i.e. the quantity $d_{\mathbf{q}}$ is real-valued and, hence, the interband interaction only shifts the equilibrium positions of ions.

Therefore, among all possible deformations of the lattice, the deformation with the components $\left|d_{\mathbf{q}}\right|$ described by Eq. (7) is characterized by the minimum of the total energy.

\section{Electronic Structure Reconstruction}

To make allowance for the band-to-band electronphonon interaction, the wave functions of the electron subsystem have to be a superposition of the Bloch wave functions for quasiparticles from both bands. Since the operator of electron-phonon coupling transforms the electron state $(\sigma, \mathbf{k})$ into the state $\left(\sigma^{\prime}, \mathbf{k}-\mathbf{q}\right)$, then, taking into account the two-band character of our model, the functions $\varphi_{1 \mathbf{k}}$ for the valence band $(\sigma=1)$ and the functions $\varphi_{2 \mathbf{k}}$ for the conduction one $(\sigma=2)$, namely,

$$
\begin{aligned}
& \varphi_{1 \mathbf{k}}=V^{-1 / 2}\left[C_{11}(\mathbf{k}) u_{1 \mathbf{k}}(\mathbf{r}) \exp (i \mathbf{k r})+\right. \\
& \left.+\sum_{\mathbf{q}} C_{12}(\mathbf{k}, \mathbf{q}) u_{2 \mathbf{k}-\mathbf{q}}(\mathbf{r}) \exp (i(\mathbf{k}-\mathbf{q}) \mathbf{r})\right]
\end{aligned}
$$

$$
\varphi_{2 \mathbf{k}}=V^{-1 / 2}\left[C_{22}(\mathbf{k}) u_{2 \mathbf{k}}(\mathbf{r}) \exp (i \mathbf{k r})+\right.
$$

$$
\left.+\sum_{\mathbf{q}} C_{21}(\mathbf{k}, \mathbf{q}) u_{1 \mathbf{k}-\mathbf{q}}(\mathbf{r}) \exp (i(\mathbf{k}-\mathbf{q}) \mathbf{r})\right]
$$


can be used as one-particle electron wave functions. In expansions (8), the Bloch amplitudes $u_{\sigma \mathbf{k}}(\mathbf{r})$ are periodic functions of the variable $\mathbf{r}$ with the period equal to the period of the crystal lattice in the disordered phase, and $V$ is the crystal volume. The Bloch wave functions, of which the functions $\varphi_{1 \mathbf{k}}$ and $\varphi_{2 \mathbf{k}}$ are constructed, are considered to be orthonormalized. Therefore, the normalization conditions impose the following constraints on the variational parameters $C_{11}, C_{12}, C_{22}$, and $C_{21}$ :

$$
\begin{gathered}
\left|C_{11}(\mathbf{k})\right|^{2}+\sum_{\mathbf{q}}\left|C_{12}(\mathbf{k}, \mathbf{q})\right|^{2}=1 \\
\left|C_{22}(\mathbf{k})\right|^{2}+\sum_{\mathbf{q}}\left|C_{21}(\mathbf{k}, \mathbf{q})\right|^{2}=1 .
\end{gathered}
$$

Suppose that electrons in the ground state occupy the levels described by the wave functions $\varphi_{1 \mathbf{k}}$, which are located only in the lower band, whereas their spins, for simplicity, have the orientation, which is the same in both bands. Then, the many-particle wave function $\psi$ of the electron subsystem, which is antisymmetric with respect to the permutations of variables, can be presented as a determinant composed of the functions $\varphi_{1 \mathbf{k}}$ and $\varphi_{2 \mathbf{k}}$ with the occupation numbers $n_{1 \mathbf{k}}=1$ and $n_{2 \mathbf{k}}=0$, respectively. Relations (8) allow new operators of creation, $\alpha_{\sigma \mathbf{k}}^{+}$, and annihilations, $\alpha_{\sigma \mathbf{k}}$, of electrons in the states described by one-particle wave functions $\varphi_{\sigma \mathbf{k}}(\mathbf{r})$ to be constructed as linear combinations of the operators $\alpha_{\sigma \mathbf{k}}^{+}$and $\alpha_{\sigma \mathbf{k}}$. For this purpose, let us equate the expansions of the electron field operator $\Psi(\mathbf{r})$ in the series in the Bloch wave functions, on the one hand, and the functions $\varphi_{\sigma \mathbf{k}}(\mathbf{r})$, on the other hand,

$V^{-1 / 2} \sum_{\sigma, \mathbf{k}} a_{\sigma \mathbf{k}} u_{\sigma \mathbf{k}} \exp (i \mathbf{k r})=\sum_{\sigma, \mathbf{k}} \alpha_{\sigma \mathbf{k}} \varphi_{\sigma \mathbf{k}}(\mathbf{r})$.

In the theory of phase transitions occurring under the influence of the interband electron-phonon interaction, the simplest case is analyzed as a rule [2], in which only one term corresponding to $\mathbf{q}=0$ is taken into account in the sum over $\mathbf{q}$ in Eq. (8). In such a manner, it is assumed that the lattice is uniformly deformed with respect to the normal coordinate, which corresponds to the mode with $\mathbf{q}=0$. This assumption is rather rough, but it allows one to make calculations considerably simpler. Below, we also use this assumption. In this case, the functions $\varphi_{1 \mathbf{k}}$ and $\varphi_{2 \mathbf{k}}$ turn out orthonormalized, provided that $\left|C_{11}(\mathbf{k})\right|^{2}+\left|C_{12}(\mathbf{k})\right|^{2}=1$. Equality (10) brings about the relations

$\left\{\begin{array}{l}a_{1 \mathbf{k}}=\alpha_{1 \mathbf{k}} C_{11}(\mathbf{k})+\alpha_{2 \mathbf{k}} C_{21}(\mathbf{k}), \\ a_{2 \mathbf{k}}=\alpha_{2 \mathbf{k}} C_{22}(\mathbf{k})+\alpha_{1 \mathbf{k}} C_{12}(\mathbf{k}) .\end{array}\right.$
Substituting Eq. (11) into Eq. (8), averaging the result using the above-indicated many-particle wave function with the occupation numbers $n_{1 \mathbf{k}}=1$ and $n_{2 \mathbf{k}}=0$, and taking into account that $\alpha_{2 \mathbf{q}}|d, \psi\rangle=0$, we obtain

$$
\langle d, \psi|\hat{H}| d, \psi\rangle=\sum_{\mathbf{k}}\left(E_{1}(\mathbf{k})+\Delta(\mathbf{k})\left|C_{12}(\mathbf{k})\right|^{2}\right)-
$$

$-\sum_{\mathbf{q}} \omega(\mathbf{q})\left|d_{\mathbf{q}}\right|^{2}$

$\left|d_{\mathbf{q}}\right|=(2 N \omega(\mathbf{q}))^{-1 / 2} \mathbf{g}_{0}(\mathbf{q}) \times$

$\times \sum_{\mathbf{k}}\left(C_{11}^{*}(\mathbf{k}) C_{12}(\mathbf{k})+C_{11}(\mathbf{k}) C_{12}^{*}(\mathbf{k})\right)$

where $\Delta(\mathbf{k})=E_{2}(\mathbf{k})-E_{1}(\mathbf{k})$. The coefficients $C_{11}$ and $C_{12}$ in formulas (12) depend only on $\mathbf{k}$. Since the vertex part $\mathbf{g}_{0}$ does not depend on the wave vectors $\mathbf{k}$, we may adopt that the coefficients $C_{11}$ and $C_{12}$ (generally speaking, complex-valued) also do not depend on the wave vector $\mathbf{k}$ or depend on it only through the corresponding phase $f$, i.e.,

$C_{11}(\mathbf{k})=\left|C_{11}\right| \exp \left\{i f_{1}(\mathbf{k})\right\}$

$C_{12}(\mathbf{k})=\left|C_{12}\right| \exp \left\{i f_{2}(\mathbf{k})\right\}$.

Therefore, taking out the absolute values $\left|C_{11}\right|$ and $\left|C_{12}\right|$ behind the summation sign over $\mathbf{k}$ in the expression for $\left|d_{\mathbf{q}}\right|$, we obtain

$$
\begin{aligned}
& N^{-1} \mathbf{g}_{0}(\mathbf{q}) \sum_{\mathbf{k}}\left\{\exp \left[i\left(f_{2}-f_{1}\right)\right]+\exp \left[i\left(f_{1}-f_{2}\right)\right]\right\}= \\
& =2 \mathbf{g}_{0}(\mathbf{q}) N^{-1} \sum_{\mathbf{k}} \cos \left(f_{2}(\mathbf{k})-f_{1}(\mathbf{k})\right) .
\end{aligned}
$$

The energy minimum corresponds to the maximum value of sum (14). Therefore, by varying the phases $f_{2}$ and $f_{1}$, we arrive at the equality $f_{2}(\mathbf{k})=f_{1}(\mathbf{k})$. Taking Eq. (14) into account, we obtain the simpler relations

$\left|d_{\mathbf{q}}\right|=2 N^{1 / 2} \mathbf{g}_{0}(\mathbf{q})(2 \omega(\mathbf{q}))^{-1 / 2}\left|C_{11}\right|\left|C_{12}\right|$,

$\langle d, \psi|\hat{H}| d, \psi\rangle=\sum_{\mathbf{k}} E_{1}(\mathbf{k})+\left(1-\left|C_{11}\right|^{2}\right) \times$ 


$$
\times \sum_{\mathbf{k}} \Delta(\mathbf{k})-\sum_{\mathbf{q}} 2 N \mathbf{g}_{0}(\mathbf{q})\left|C_{11}\right|^{2}\left|C_{12}\right|^{2} .
$$

Now, we can proceed to finding the minimum of energy (12) with respect to the parameters $\left|C_{11}\right|$ and $\left|C_{12}\right|$. Since those parameters are coupled with each other by the normalization condition, actually we have a single parameter to vary.

We confine the consideration to the case of a uniform deformation, $\mathbf{g}_{0}(\mathbf{q})=\mathbf{g}_{0} \cdot \delta_{\mathbf{q} 0}$. It corresponds to the well-known interband theory of ferroelectric phase transitions, which analyzes the behavior of a "soft" vibration mode under the influence of the interaction with electrons. Then, using the coefficient $\left|C_{12}\right|$ as a variational variable, on the basis of Eq. (12), we obtain

$\left|C_{12}\right|^{2}=0,25\left(2 \mathbf{g}_{0}^{2}-\bar{\Delta}\right) \mathbf{g}_{0}^{2}$,

$\left|C_{11}\right|^{2}=0,25\left(2 \mathbf{g}_{0}^{2}+\bar{\Delta}\right) \mathbf{g}_{0}^{2}$,

where $\bar{\Delta}=N^{-1} \sum_{\mathbf{k}} \Delta(\mathbf{k})$. So, the energy minimum turns out to equal

$$
\langle d, \psi|\hat{H}| d, \psi\rangle_{\min }=\sum_{\mathbf{k}}\left\{E_{1}(\mathbf{k})-\left(2 \mathbf{g}_{0}^{2}-\bar{\Delta}\right)\left(8 \mathbf{g}_{0}^{2}\right)^{-1}\right\} .
$$

The deformation parameter, which corresponds to this minimum, equals

$\left|d_{0}\right|=N^{1 / 2}\left(8 \omega(0) \mathbf{g}_{0}^{2}\right)^{-1 / 2} \sqrt{4 \mathbf{g}_{0}^{4}-\bar{\Delta}^{2}}$.

A condition for the structural reconstruction of the lattice to take place is, in this case, the condition of strong coupling,

$2 \mathbf{g}_{0}^{2}>\bar{\Delta}$

at which the square of the absolute value $\left|C_{12}\right|^{2}$ really turns out positive. This condition was obtained in work [2] dealing with the interband theory of ferroelectric transitions accompanied by a vibration mode "softening". However, in the cited work, the inequality looked like $4 \mathbf{g}_{0}^{2}>\bar{\Delta}$, i.e. the coefficient was equal to 4 rather than 2 , because the authors supposed the existence of electrons with two spin orientations in both the valence and conduction bands, whereas we take into consideration only one spin orientation for simplicity. In the case of work [2], i.e. at two possible spin orientations, the quantity $2 \mathbf{g}_{0}^{2}$ in relations (17) and (18) should be substituted by $4 \mathbf{g}_{0}^{2}$, and the quantity $4 \mathbf{g}_{0}^{4}$ in relation (19) by $16 \mathbf{g}_{0}^{4}$.

\section{Conclusion}

To summarize, the method of varying the states of electron and phonon subsystems at the strong interband electron-phonon coupling enabled a relationship between a lattice deformation at $T \rightarrow 0$, which arises owing to the phase transition, and a modification of the electron distribution function in a unit cell owing to the hybridization between the electron states in the conduction and valence bands to be established. The obtained strong-coupling criterion coincides with that for the frequency zeroing of the "soft" mode [2] at $T=T_{c}$, where $T_{c}$ is the temperature of a phase transition of the second kind (the displacive phase transition). According to the results of work [2], $T_{c} \approx$ $4 \mathbf{g}_{0}^{2}-\bar{\Delta}$. This relation allows us to determine that $\mathrm{g}_{0}^{2} \approx 1.01 \mathrm{eV}$ at $\bar{\Delta} \approx 4 \mathrm{eV}$ (e.g., for crystals with a wide energy gap) and $T_{c} \approx 400 \mathrm{~K}$ (e.g., for SbSI). For the spin configuration considered in this work, $2 \mathbf{g}_{0}^{2}-$ $\bar{\Delta} \approx 0.01 \mathrm{eV}$ for a SbSI crystal. Hence, we may assert that every sublevel in the valence band, in accordance with Eq. (18), becomes shifted downward by the value

$\delta E \equiv \frac{\left(2 \mathbf{g}_{0}^{2}-\bar{\Delta}\right)^{2}}{8 \mathbf{g}_{0}^{2}}=\frac{T_{c}^{2}}{4 \bar{\Delta}}$

$\delta E=10^{-4} \cdot \frac{\bar{\Delta}}{4} \approx 10^{-4} \mathrm{eV}$.

In other words, after the crystal deformations at $T \rightarrow 0$ having finished, the crystal energy, owing to the interaction between the electrons and the deformation with respect to the "soft"-mode normal coordinate, diminishes by an order of magnitude of $10^{-4} \mathrm{eV}$ per one crystal cell.

The temperature $T_{c}>0$ is evidently possible only for those transverse modes of vibrations of the medium, for which

$4 \mathbf{g}_{0}^{2}(\mathbf{q})>\bar{\Delta}(\mathbf{q})$.

Bearing in mind that the wave-vector set for phonon modes is almost continuous even in crystals of millimeter dimensions, we can be sure that, if inequality (22) is satisfied for a certain single mode, it will also hold true for many unstable modes close to it. For those modes, for which inequality (22) transforms into the equality, the critical temperature $T_{c}=0$. Therefore, we may suppose that, in the temperature range from 0 to $T_{c \max }$, a series of phase transitions have to 
take place, which are associated with the crystal deformation with respect to the normal coordinates of all modes in the indicated series. As a result, the translational symmetry of the crystal turns out violated. It is evident that, at $T \rightarrow 0$, a domain structure should be formed, with the domain size being determined by the width of the unstable mode interval in the Brillouin zone. Of course, the size of domains is also affected by the degree of crystal imperfection.

In other words, the considered model of phase transitions occurring under the influence of the interband electron-phonon interaction describes a smeared phase transition of the second kind. The smearing width depends on the temperature interval, in which the deformation with respect to the normal coordinates of the majority of unstable modes takes place. Since the model with Hamiltonian (1) has been constructed on the basis of the assumption that deformations with respect to the normal coordinates of different modes are independent, the corresponding conclusions are not valid for substances, in which those modes interact with one another directly or by means of modes belonging to other branches of crystal lattice vibrations. If the coupling between modes - e.g., the striction ones - does take place, the deformation with respect to the normal coordinates of a certain mode set can occur in the form of a single jump without zeroing of the frequencies of all those modes, i.e. as a phase transition of the first kind.

It is of interest to analyze microprocesses, the sequence of which results in a FT2. The FT2 begins from the ground state $|0\rangle$ of a highly symmetric phase, which starts to change stochastically under the influence of ordering processes. A possibility of ordering fluctuations follows from the identity of equalities

$|0\rangle=|I| 0\rangle=U^{+} U|0\rangle$,

where $I$ is the unit transformation operator for the initial vector of state $|0\rangle$. The operator $I$ can be presented as a product of the Hermitian conjugate unitary operators $U^{+}$and $U$, one of which, $U$, introduces a coherent deformation existing in some section of the crystal into the structure of the ground state $|0\rangle$. Hence, there emerges a new ground state $U|0\rangle$, in which the electrons become redistributed adiabatically in every unit cell of the deformed section, so that the deformation energy becomes lower. The deformed state of the type $U^{+}|0\rangle$ is deformed with the opposite sign; this deformation is not accompanied by a reduction of the interaction energy as the electrons are redistributed in the cell (as it was in the case of an $U|0\rangle$-type deformation). Therefore, if the redistribution of the electron density diminishes the energy of a deformation of the type $U|0\rangle$, it increases the energy of a deformation of the type $U^{+}|0\rangle$. As a result, the deformation $U|0\rangle$ becomes quasiequilibrium, and the deformation $U^{+}|0\rangle$ is nonequilibrium and, therefore, decays rapidly. In such a manner, the sections with a deformation of the type $U|0\rangle$ are accumulated in the crystal, and this deformation becomes equilibrium as the temperature falls down to $T=T_{c}$. The decay of a nonequilibrium deformation even before the FT2 takes place can manifest itself in the form of pulses similar to Barkhausen pulses emerging at the repolarization of a ferroelectric material [7-9]. The probability $W(n)$ for $n$ such pulses to emerge from $n$ phonons was calculated in the framework of the theory of coherent deformations [10]. If a deformation is formed, on the average, from $\bar{n}$ phonons,

$W(n)=\frac{\bar{n}^{n} \exp (-\bar{n})}{n !}$.

Therefore, before the structural reconstruction in the crystal at the FT2 has come to the end, pulses with various energies obeying the Poisson distribution (formula (24)) can be observed in it as FT2 forerunners. Those pulses, which evidence a decay of the state $U^{+}|0\rangle$ into stationary states with definite quantum numbers appear, on the average, before the transition $|0\rangle \rightarrow U|0\rangle$ takes place. It is so, because their appearance is induced by the formation of an ordering deformation in the crystal and its separation from the nondeformed state. If the system is in the range of an ordered phase and is heated up from $T=0$, the pulses do not arise, because the structure cannot be reconstructed until the temperature becomes equal to the transition one, whereas the equilibrium structure at temperatures above $T_{c}$ is represented by the disordered phase. Therefore, the deformation, which took place in the ordered phase, decays after the temperature has crossed $T_{c}$. The energy released at that is identical to that released at the transition from the highly symmetric phase into the ordered one.

1. V.L. Bonch-Bruevich, Usp. Fiz. Nauk 56, 56 (1955).

2. E.V. Bursian and Ya.G. Girshberg, Coherent Effects in Ferroelectrics (Prometei, Moscow, 1989) (in Russian).

3. A.E. Myasnikova, E.N. Myasnikov, and Z.P. Mastropas, Teor. Mat. Fiz. 157, 1595 (2008).

4. L. Onsager, Phys. Rev. 65, 117 (1944). 
5. L.D. Landau and E.M. Lifshitz, Statistical Physics, Part 1 (Pergamon Press, Oxford, 1980).

6. M. Lax, in Statistical Physics, Phase Transitions and Superfluidity, edited by M.P. Chretien, E.P. Gross, and S. Deser (Gordon and Breach, New York, 1968), v. 2, p. 271.

7. A.E. Poladino, J. Am. Ceram. Soc. 48, 476 (1965).

8. A.E. Poladino, L.G. Rubin, and J.S. Waugh, J. Phys. Chem. Sol. 26, 391 (1965).

9. J.F. Schooley, W.R. Hosler, and M.L. Cohen, Phys. Rev. Lett. 12, 474 (1964).

10. J.R. Klauder and E.C.G. Sudarshan, Fundamentals of Quantum Optics (Benjamin, New York, 1968).

Received 05.07.11.

Translated from Russian by O.I. Voitenko

\section{МІКРОПРОЦЕСИ ПРИ ФАЗОВИХ ПЕРЕХОДАХ

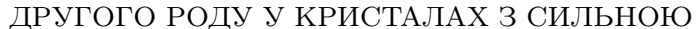 МІЖЗОННОЮ ЕЛЕКТРОН-ФОНОННОЮ ВЗАЕМОДІЕЮ}

\section{Е.Н. Мясников, З.П. Мастропас}

$\mathrm{P}$ е $з$ ю м е

У роботі теоретично розглянуто систему сильно взаємодіючих електронів і фононів у кристалі, яка зазнає при зміні температури перетворень, еквівалентних фазовому переходу другого роду зі зміщеннями положень рівноваги атомів. Показано, що розклади термодинамічного потенціалу за параметром порядку, прийняті у феноменологічній теорії Ландау, можуть приводити до положення рівноваги, що не відповідають якомунебудь реальному стану кристала. Показано також, що при фазових переходах з пониженням чи підвищенням температури стрибками виділяється енергія деформації у вигляді імпульсів типу імпульсів Баркгаузена. 